\title{
Indicadores altmétricos da produção de pesquisadores brasileiros: análise dos bolsistas de produtividade do CNPq
}

\author{
Ronaldo Ferreira Araujo \\ Universidade Federal de Alagoas, Instituto de Ciências Humanas, Comunicação e Artes, \\ Programa de Pós-Graduação em Ciência da Informação, Maceió AL, Brasil \\ ronaldo.araujo@ichca.ufal.br
}

Marcelo Alves

Universidade Federal Fluminense, Instituto de Artes e Comunicação Social, Programa de Pós-Graduação em Comunicação, Niterói, RJ, Brasil

marceloalves.ufsj@hotmail.com

DOI: https://doi.org/10.26512/rici.v12.n3.2019.17203

Recebido/Recibido/Received: 2018-10-16

Aceitado/Aceptado/Accepted: 2019-08-08

Resumo: $O$ presente trabalho analisa o desempenho altmétrico de publicações brasileiras cujos autores recebam bolsas de produtividade em pesquisa (PQ) concedidas pelo Conselho Nacional de Desenvolvimento Científico e Tecnológico (CNPq). Foram considerados para o universo da pesquisa os pesquisadores cujas bolsas de PQ estavam vigentes em outubro de $2017(n=14.609)$. A produção científica registrada no Currículo Lattes desses autores foi coletada pelo GetLattesData, pacote da linguagem R, e filtrada por artigos de periódicos acadêmicos publicados entre 2016 e outubro de 2017 que possuíssem o Digital Object Identifier $(\mathrm{n}=99064)$. Os dados de atenção online foram analisados segundo sua distribuição por densidade e variação; idioma de publicação e área de conhecimento dos artigos. $O$ desempenho médio por tipo de fonte dos dados altmétricos também foi avaliado. A maior parte dos artigos não apresentou indicadores de atenção online e bem poucos revelaram índices elevados. 0 melhor desempenho na atenção online foi verificado nas publicações em inglês das áreas de Ciências da Saúde e Ciências Biológicas, com destaque para o Mendeley e Twitter como fontes do maior número de menções.

Palavras-chave: Altmetria. Atenção online. Bolsista de produtividade. CNPq. Impacto social.

Indicadores altmétricos de la producción de investigadores brasileños: análisis de los becarios de productividad del CNPq

Resumen: El presente trabajo analiza el desempeño altmétrico de publicaciones brasileñas cuyos autores reciben becas de productividad en investigación ( $\mathrm{PQ}$ ) concedidas por el Consejo Nacional de Desarrollo Científico y Tecnológico (CNPq). Se consideraron para el universo de la investigación los investigadores cuyas bolsas de PQ estaban vigentes en octubre de 2017 ( $n=14.609)$. La producción científica registrada en el Currículo Lattes de estos autores fue recolectada por el GetLattesData, paquete del lenguaje R, y filtrada por artículos de revistas académicas publicadas entre 2016 y octubre de 2017 que poseía el Digital Object Identifier $(n=99064)$. Los datos de atención en línea se analizaron según su distribución por densidad y variación; idioma de publicación y área de conocimiento de los artículos. El rendimiento promedio por tipo de fuente de los datos de la altmetría también fue evaluado. La mayor parte de los artículos no presentó indicadores de atención en línea y muy pocos revelaron índices elevados. El mejor desempeño en la atención en línea fue verificado en las publicaciones en inglés de las áreas de Ciencias 
de la Salud y Ciencias Biológicas, con destaque para Mendeley y Twitter como fuentes del mayor número de menciones.

Palabras clave: Altmetría. Atención en línea. Beca de productividad. CNPq. Impacto social.

Altmetrics of scholarly publications of Brazilian researchers: analysis of the CNPq productivity holders

Abstract: This paper aims to analyses the altmetric performance of Brazilian publications authored by researchers who are holders of productivity scholarship (PQ) of the National Council of Scientific and Technological Development (CNPq). The scope of this research assesses the PQs in activity on October, 2017 ( $n=14.609$ ). The collection of scientific production registered on Lattes was conducted via GetLattesData and filtered by articles from academic journals published between 2016 and October 2017 that hold the Digital Object Identifier $(n=99064)$. The online attention data are analyzed according to their distribution by density and variation; language of the publication and field of knowledge; and by average performance of the type of source that has provided its altmetric values. The result show long tail behavior of the altmetric score, with most part of the articles with no online attention, while few articles have a high index. The average of the online attention indicates a better performance of articles written in English and belonging to the Health and Biological Sciences field of knowledge. As for the sources, there was a good performance from Mendeley followed by Twitter.

Keywords: Altmetrics. Online Attention. Brazilian productivity holder. CNPq. Social impact.

\section{Introdução}

A economia da atenção tem sido considerada uma abordagem adequada à gestão de informações, pois considera a atenção humana como uma mercadoria escassa, sendo necessária a aplicação da teoria econômica para resolver os problemas de gerenciamento informacional. Essa perspectiva coloca a atenção como recurso estratégico da ação humana frente ao crescimento abundante e imediato de conteúdos disponíveis, que se torna um fator limitante no consumo de informações.

Para Davenport e Beck (2001) a atenção é o engajamento mental focado em um determinado item de informação. Os itens entram em nossa consciência e dentre eles atendemos a um item específico sobre o qual decidimos agir. Assim, a atenção é usada para filtrar a informação mais importante a partir de um conjunto de informações que envolvem o ser humano, e que se proliferam cada vez mais nas redes e mídias sociais dos ambientes digitais.

No contexto da comunicação científica e do reconhecimento da necessidade de filtros que auxiliem os pesquisadores a decidir quando se dedicar a um item em detrimento de outro - uma vez que "ninguém pode ler tudo" - Priem et al. (2010), em seu manifesto, ressaltam a necessidade de mais ferramentas e pesquisas baseadas em altmetria.

A altmetria tem sido considerada o campo de estudos que se dedica ao uso de dados cibermétricos para análises cientométricas (GOUVEIA, 2017) e nasce com a possibilidade de mensuração rápida de indicadores da atenção online que produtos da ciência alcançam, logo após sua publicação. Assim, os indicadores expressam a atenção online por meio da disseminação e outros tipos de interação que tais produtos geram na web, atingindo não apenas 
os interessados dentro da comunidade científica, mas também o grande público - cuja apropriação indica impacto social.

O presente trabalho procura analisar o desempenho altmétrico de publicações brasileiras que tenham como autores pesquisadores bolsistas de produtividade em pesquisa (PQ) do Conselho Nacional de Desenvolvimento Científico e Tecnológico (CNPq). As bolsas PQs são destinadas a pesquisadores de todas as áreas que se destaquem entre seus pares, valorizando sua produção científica segundo critérios normativos (CNPq, 2017), baseados não só na qualidade do projeto submetido, mas também na "qualidade" do pesquisador (WAINER; VIEIRA, 2013).

Algumas pesquisas se dedicaram à avaliação da produção científica resultante de pesquisas financiadas com bolsas de produtividade, mas o foco geralmente é voltado para a análise de métricas tradicionais e medidas bibliométricas (WAINER; VIEIRA, 2013; FONSECA; DIGIAMPIETRI, 2016). São necessários estudos que, além do impacto acadêmico que recebem, a partir das citações, avaliem a mesma produção na perspectiva da atenção online que tais pesquisas despertam dentro e fora da comunidade científica, tema em que ainda há poucos trabalhos publicados, mesmo na literatura internacional (WANG, X. et al., 2016).

O artigo discorre brevemente sobre a altmetria para avaliação de pesquisas em nível nacional por meio de alguns estudos. Em seguida detalha os procedimentos metodológicos adotados. Por fim discute os resultados e expressa considerações finais.

\section{Altmetria para avaliação de pesquisa em nível nacional: algumas considerações}

O número crescente de cientistas e pesquisadores compartilhando seus artigos em mídias sociais como Facebook e Twitter e discutindo seus resultados de pesquisa on-line com membros da academia e público em geral tem reforçado a emergência dos estudos da altmetria.

Consideradas complementares aos tradicionais indicadores bibliométricos de citação, as métricas alternativas ainda contam com poucos estudos que tenham examinado a relação entre produtividade acadêmica e o desempenho altmétrico em nível nacional (ALHOORI et al, 2014; THELWALL; MAFLAHI, 2014; WANG et al, 2016; CHO, 2017; PARK; PARK, 2018). Além disso, não está claro se a altmetria pode ser considerada uma ferramenta de medição universal, já que o acesso à Internet e o uso de ferramentas de mídia social variam de um país ou região para outro (ALHOORI et al, 2014; WANG et al, 2016), assim como seu idioma (ALPERIN, 2013; THELWALL; MAFLAHI, 2014).

Alhoori e outros (2014) investigaram se as altmetrias podem apoiar a avaliação da pesquisa em vários países e verificaram como os valores altmétricos diferem quando analisados nos níveis de artigo e de país. Os autores selecionaram 35 países desenvolvidos e em 
desenvolvimento que publicaram até 2.000 artigos indexados na Scopus por ano, de 10 de janeiro de 2010 a 5 de junho de 2014. Foram incluídos na pesquisa os artigos co-escritos por pesquisadores de diferentes países e, de acordo com os resultados dos autores, verificou-se que a altmetria pode apoiar a avaliação de pesquisas para todos os países estudados.

Thelwall e Maflahi (2014) avaliaram até que ponto as afiliações nacionais dos autores de artigos afetam as afiliações nacionais de seus leitores no Mendeley. Com base em artigos em inglês da Web of Science em dez campos da ciência, envolvendo medicina, ciências sociais e humanidades, os resultados de modelos estatísticos comparando afiliações de autores e leitores sugerem que, na maioria dos campos, os usuários do Mendeley leem desproporcionalmente mais artigos de autores de seu próprio país (THELWALL; MAFLAHI, 2014). Além disso, há vários casos em que usuários de determinados países tendem a ignorar artigos de outros países específicos, embora não esteja claro se isso reflete tendências nacionais ou diferentes especialidades nacionais dentro de um campo (THELWALL; MAFLAHI, 2014).

Ao refletirem sobre fatores que influenciam a atenção online que uma publicação científica recebe nas mídias sociais, Wang e outros (2016) constataram que poucos estudos analisaram o impacto da nacionalidade do autor no desempenho altmétrico. Essa constatação os motivou a investigarem o desempenho altmétrico de publicações na área de Biotecnologia e Microbiologia Aplicada publicadas por autores chineses. Os autores verificaram que artigos publicados por autores de afiliações chinesas têm uma visibilidade muito menor na web social do que artigos de autores nascidos em outros países, tendo menor número de publicações circulando no Twitter, e as poucas que circulam atraem menos atenção social. Para os autores esta situação desfavorável para os chineses decorre, em parte, da inacessibilidade das principais plataformas de redes sociais na China continental (WANG et al, 2016).

Cho (2017) analisou o impacto de artigos de pesquisa coreanos de ciências médicas, engenharia, ciências sociais, artes e humanidades por meio da altmetria, comparando seu desempenho com indicadores tradicionais da bibliometria. A frequência de artigos mencionados no Twitter mostrou-se mais alta nas ciências médicas do que em outros campos. Por outro lado, a frequência de artigos no Mendeley mostrou-se mais alta nas ciências sociais e nas artes e humanidades do que em outros campos. Após a análise comparativa entre desempenho de indicadores altmétricos e bibliométricos, verificou-se que existe uma correlação positiva entre o número de artigos que foram salvos no Mendeley e citados. Segundo a autora, estudos de altmetria podem auxiliar a identificar o impacto invisível de sua produção de pesquisa na sociedade global e, ao encontrar o campo acadêmico que está recebendo atenção, os pesquisadores poderiam prospectar o impacto geral da pesquisa no país e utilizá-lo para criar um plano de pesquisa e desenvolvimento (CHO, 2017). 
Na pesquisa de Park e Park (2018) a influência de artigos acadêmicos em cinco países asiáticos foi avaliada de acordo com o desempenho de indicadores altmétricos. Em particular, os autores examinaram menções altmétricas de artigos acadêmicos em mídias sociais analisando os tipos de mídia social em que os artigos foram mencionados com maior frequência segundo as disciplinas. Quanto aos indicadores altmétricos por país, o Japão teve o melhor desempenho, seguido pela China, Coréia do Sul, Taiwan e Cingapura, cujos artigos foram mais frequentemente mencionados na plataforma Mendeley, seguida pelo Twitter e Facebook, com destaque para os campos da saúde, medicina e ciências (PARK; PARK, 2018).

$\mathrm{O}$ traço comum aos estudos que foram brevemente descritos aqui foi a constatação da necessidade de mais estudos para aferir as métricas alternativas de avaliação das pesquisas nacionais (isoladas e comparadas), aumentando o número de países e artigos analisados e qualificando o emprego de correlações estatísticas que permitem melhor compreender o fenômeno. É preciso observar que, por vezes, os resultados podem estar indicando tendências que se aplicam a campos e países como sugestão de preferência nacional, embora talvez não universal (THELWALL; MAFLAHI, 2014).

\section{Procedimentos metodológicos}

O banco de dados dessa pesquisa foi composto por uma amostragem de pesquisadores brasileiros, identificados por uma aplicação web scraping, com extração de informações, junto ao site do CNPq, de todos os cientistas que possuem bolsa de Produtividade em Pesquisa (PQ) vigente no Brasil em outubro de 2017, resultando em um total de 14.609 pesquisadores. Em seguida foram extraídas e tabuladas suas publicações registradas no Currículo Lattes, a partir do pacote GetLattesData (PERLIN, 2017), da linguagem estatística R. Uma vez que publicações recentes costumam apresentar maior desempenho altmétrico, foram selecionados os artigos de periódicos acadêmicos publicados entre 2016 e outubro de 2017 que possuíssem o Digital Object Identifier (DOI) informado no Lattes, o que correspondeu a 99.064 artigos. Por fim, foram excluídos DOI duplicados, referentes a artigos em coautoria, resultando em uma amostra final de 69.419 artigos. Para artigos em coautoria com mais de um bolsista PQ considerou-se relevante a ordem de autoria no trabalho, contabilizando-se apenas a área de conhecimento do primeiro.

A extração de dados da atenção online dessa produção foi realizada pelo pacote rAltmetric (rOpenSci, 2017) que consulta a Application Programming Interface (API) pública do Altmetric a partir do DOI das publicações e retorna uma planilha com os dados produzidos pela plataforma. Em seguida, os metadados fornecidos pelo CNPq (filiação, nível da bolsa PQ, Grande 
Área) e pelo Lattes (Idioma) foram cruzados, para produzir uma tabela única com as informações de interesse do trabalho.

O Attention Score Altmetric é uma medida geral da atenção que um artigo ou conjunto de dados tem recebido online, e deve refletir a visibilidade (alcance) e a influência e engajamento (qualidade da atenção, local postado e reputação de quem posta) que pode variar de acordo com a fonte ou tipo de mídia considerada (ALTMETRIC, 2017). Vale lembrar que os agregadores da Altmetric acompanham as menções de um determinado artigo nas várias plataformas de forma automatizada para derivar seu valor numérico composto como uma medida em tempo real do impacto online, que é sempre apresentada como um número inteiro.

Esta pontuação reflete um total ponderado das menções do artigo pelas várias plataformas online, pelo qual pesos são atribuídos pela Altmetric para refletir o alcance relativo antecipado de vários tipos de fontes (por exemplo, pesos padrão de 8 para um post de notícias, 5 para um postagem de blog, 1 para o Twitter, 3 para um documento de política ou postagem da Wikipédia e 0,25 para o Facebook) (ALTMETRIC, 2017). Essas escolhas têm recebido algumas críticas por parte da comunidade científica, seja por não ficar claro o porquê a Altmetric decidiu incluir certas mídias sociais, mas excluir outras, como os leitores de Mendeley, uma fonte de mídia social exaustiva e prevalente para os cientistas (ZAHEDI; COSTAS; WOUTERS, 2014) e principalmente quanto aos pesos atribuídos às mídias sociais selecionadas, que não foram validados teoricamente ou empiricamente. Eles parecem arbitrários ou simplesmente baseados nas crenças da equipe da Altmetric e os documentos online não fornecem os algoritmos para agregação, apenas uma breve descrição (HUANG; WANG; WU, 2018).

Ainda assim, a Altmetric tem se consolidado como a principal provedora de dados altmétricos. Na presente pesquisa os dados de atenção online foram analisados segundo sua distribuição por densidade e variação; idioma da publicação e área de conhecimento dos pesquisadores; e por desempenho da média por tipo de fonte que forneceu seus valores altmétricos.

\section{Resultados e discussão}

As soluções disponíveis para monitoramento e análise da atenção online e dos dados altmétricos da produção científica exigem dessa produção padrões de identificação, como o DOI, que garantam, entre outras vantagens, sua distinção. Foi verificado que $73,41 \%$ das publicações analisadas possui o identificador, o que parece revelar que os bolsistas PQ estão atentos a publicar seus resultados de pesquisa em periódicos com este padrão.

A distribuição da variável score (atenção online) segundo sua densidade e variação pode ser vista na Figura 1, ambas transformados em escala logarítmica. No gráfico à esquerda, 
percebe-se que a densidade evidencia o comportamento de cauda longa da variável, isto é, a maior parte dos artigos, concentrada à esquerda da curva de densidade, não possui atenção online, ou seja, seu score altmetrics é igual a zero, enquanto bem poucos artigos possuem um score elevado. No gráfico à direita, o score altmétrico não mudou muito a distribuição, tendo em vista o número muito elevado de zeros. A escala foi mantida a mesma em ambos os gráficos para não quebrar a distribuição e garantira não distorção dos dados.

Figura 1. Distribuição da atenção online por graus de densidade e variação

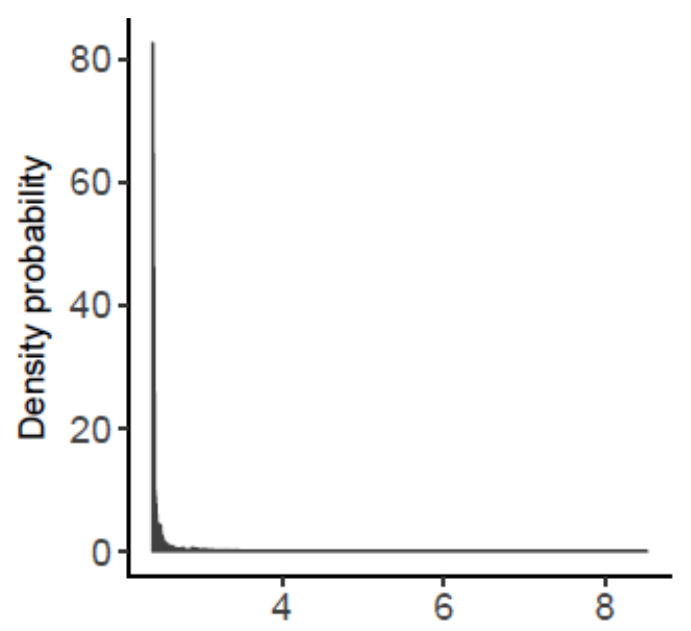

Altmetrics score (log transformed)

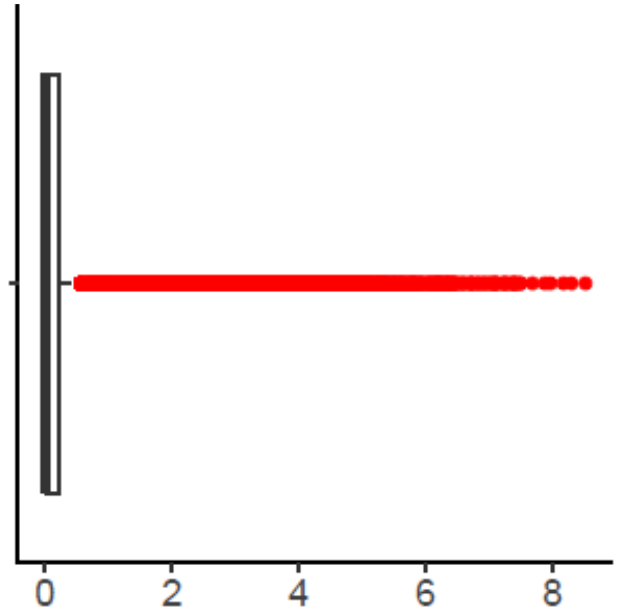

Altmetrics score (log transformed)

Fonte: dados da pesquisa (2017)

No segundo gráfico, que reforça o comportamento do primeiro, os dados mostram que todas as medidas de posição estão próximas de 0 e a variável score possui um número muito grande de outliers, valores extremos que se afastam muito da mediana, indicados pelos pontos vermelhos no gráfico, variando de 0 a 4966.648. A distribuição da média da atenção online por idioma e sua porcentagem por tipo de fonte podem ser verificadas a seguir, nos Gráficos 1 e 2. 
Gráfico 1. Média da atenção online por idioma

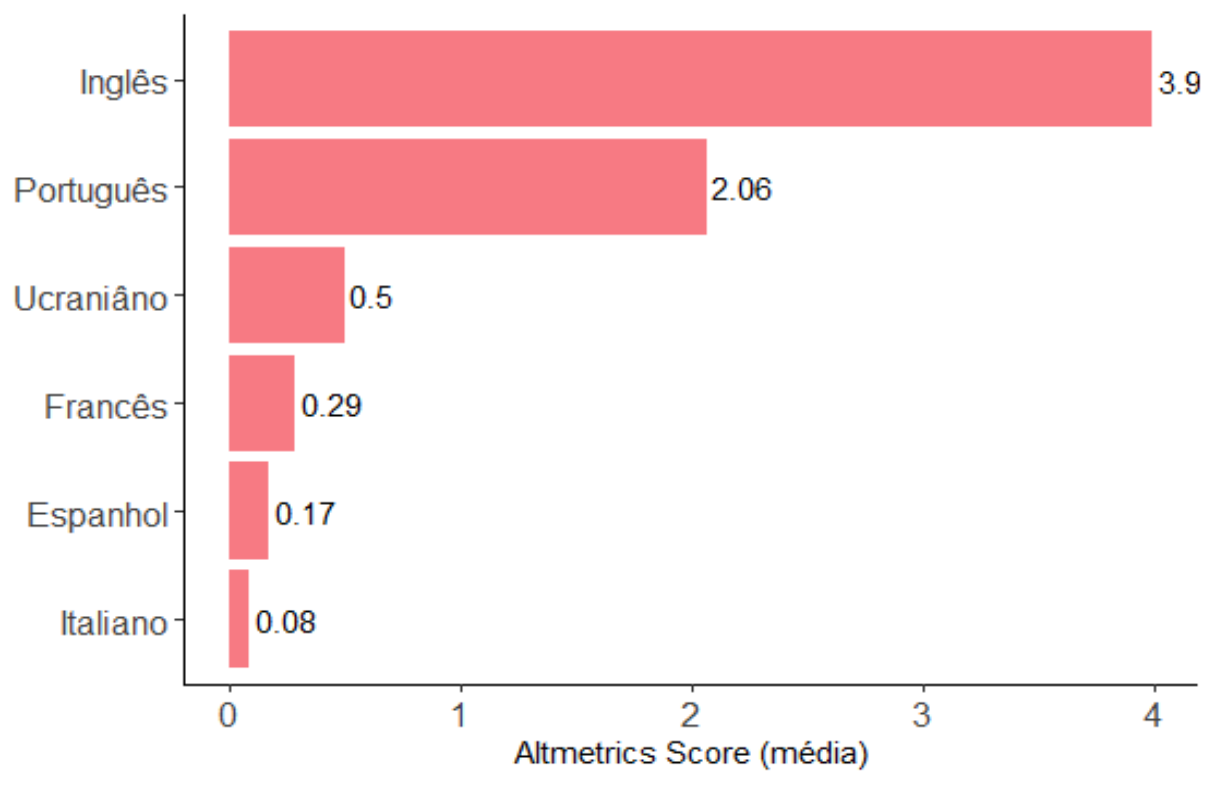

Fonte: dados da pesquisa (2017)

Tabela 1. Porcentagem dos valores altmétricos por idioma e tipo de fonte

\begin{tabular}{|l|l|l|l|l|l|}
\cline { 2 - 6 } \multicolumn{1}{c|}{} & Fontes & \multicolumn{4}{l|}{} \\
\hline Idioma & Twitter & Facebook & Mendeley & Imprensa & Blogs \\
\hline Espanhol & $0.01 \%$ & $0.02 \%$ & $0.01 \%$ & $0.00 \%$ & $0.00 \%$ \\
\hline Inglês & $88.40 \%$ & $83.60 \%$ & $86.98 \%$ & $87.40 \%$ & $84.09 \%$ \\
\hline Outro & $0.00 \%$ & $0.02 \%$ & $0.01 \%$ & $0.00 \%$ & $0.03 \%$ \\
\hline Português & $11.59 \%$ & $16.36 \%$ & $13.00 \%$ & $12.60 \%$ & $15.88 \%$ \\
\hline
\end{tabular}

Fonte: dados da pesquisa (2017)

Na contramão da noção de democratização do conhecimento científico atribuído às mídias sociais, algumas pesquisas mostram que muitas vezes a análise de seu uso reproduz as mesmas disparidades de visibilidade das métricas tradicionais, como o fato das publicações de países emergentes tenderem a ter menor visibilidade nas plataformas de redes sociais (ALPERIN, 2015) e do inglês ser a língua dominante (FAUSTO et al., 2012) e ter maior cobertura e melhor desempenho (ALPERIN, 2013). A representação da média da atenção online por idioma, no Gráfico 1, e da porcentagem dos idiomas por tipo de fonte, no Gráfico 2, confirmam tal comportamento.

De acordo com Alperin (2013) o futuro da comunicação acadêmica precisa incluir diversos contextos locais do mundo em desenvolvimento e ser adaptado para atender às metas nacionais de desenvolvimento. Para o autor, as fontes consultadas por ferramentas altmétricas devem, por exemplo, incluir bancos de dados não ingleses e menções em mídias sociais e em 
serviços como a Wikipédia devem consultar todos os idiomas disponíveis, não apenas o inglês (ALPERIN, 2013).

A distribuição dos dados de atenção online por área de conhecimento (Gráfico 3) e seu comparativo por tipo de idioma (Tabela 2) também foram observados, tendo em vista que caracterizam o desempenho da produção analisada e podem reforçar o mesmo comportamento percebido quanto ao idioma e tipo de fonte.

Gráfico 2. Média da atenção online por área de conhecimento

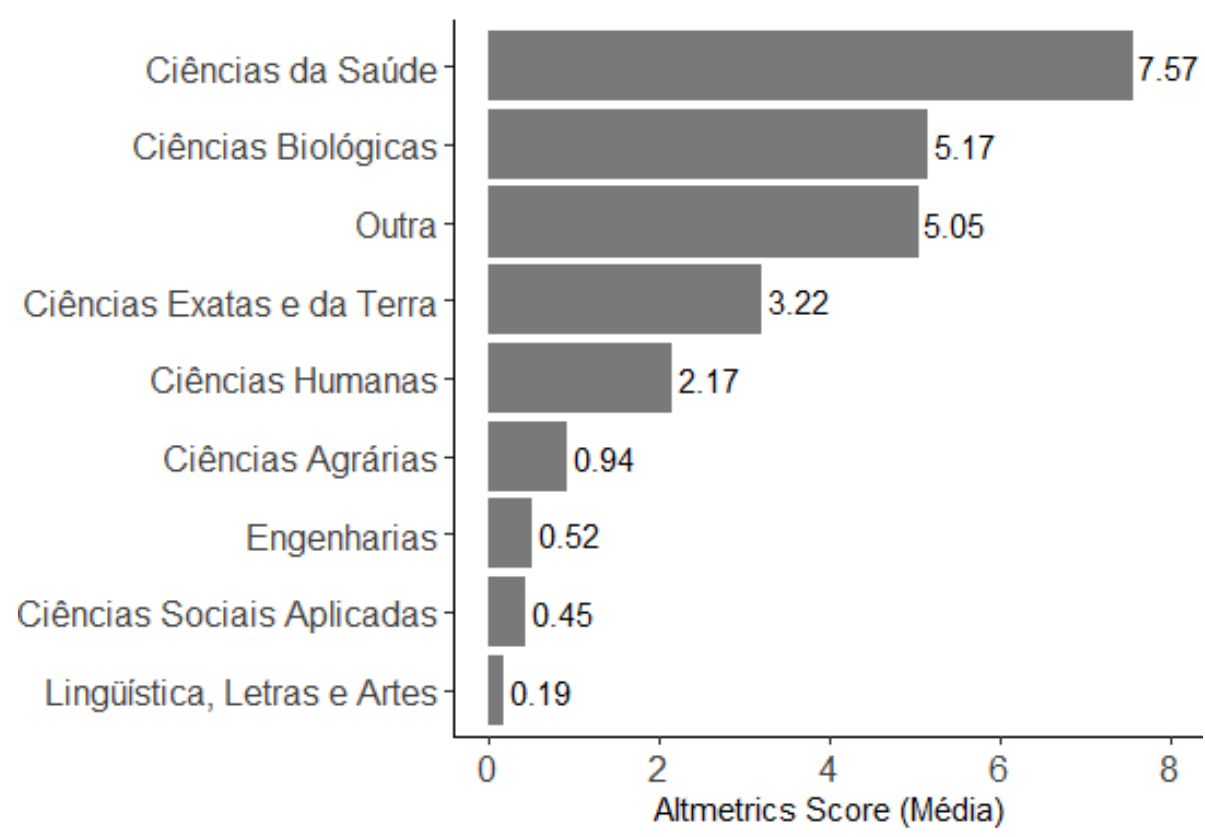

Fonte: dados da pesquisa (2017)

Tabela 2. Porcentagem da atenção online (Altmetrics Score) por área de conhecimento

\begin{tabular}{|l|l|l|l|l|}
\cline { 2 - 5 } \multicolumn{4}{l|}{} & \multicolumn{4}{l}{ Idioma } \\
\hline Grande Área & Espanhol & Inglês & Outro & Português \\
\hline Ciências Agrárias & $0.0 \%$ & $73.2 \%$ & $0.0 \%$ & $26.8 \%$ \\
\hline Ciências Biológicas & $0.0 \%$ & $84.4 \%$ & $0.0 \%$ & $15.6 \%$ \\
\hline Ciências da Saúde & $0.2 \%$ & $81.6 \%$ & $0.1 \%$ & $18.2 \%$ \\
\hline Ciências Exatas e da Terra & $0.0 \%$ & $83.7 \%$ & $0.0 \%$ & $16.2 \%$ \\
\hline Ciências Humanas & $0.7 \%$ & $45.3 \%$ & $0.4 \%$ & $53.6 \%$ \\
\hline Ciências Sociais Aplicadas & $0.2 \%$ & $50.1 \%$ & $0.3 \%$ & $49.3 \%$ \\
\hline Engenharias & $0.1 \%$ & $80.6 \%$ & $0.0 \%$ & $19.3 \%$ \\
\hline Linguística, Letras e Artes & $0.3 \%$ & $36.2 \%$ & $0.6 \%$ & $63.0 \%$ \\
\hline Outra & $0.0 \%$ & $77.0 \%$ & $0.1 \%$ & $22.9 \%$ \\
\hline
\end{tabular}

Fonte: dados da pesquisa (2017) 
Quanto ao Gráfico 2, a média da atenção online indica melhor desempenho das Ciências da Saúde, com 7.56 e das Ciências Biológicas, com 5.17 pontos. O resultado é similar ao do estudo de Costas, Zahedi e Wouters (2014) no qual as Ciências da Saúde e as Ciências Biológicas também se apresentam como áreas do conhecimento que obtiveram maior atenção online, com valores altmétricos superiores às demais. Mas no geral, os valores obtidos pela produção dos pesquisadores brasileiros são muito baixos.

A Tabela 2 confirma o fato de o idioma influenciar o desempenho das publicações uma vez que exatamente as áreas que mais publicam em português são as que menos obtêm atenção online ou que apresentam valores mais baixos desta atenção.

O Attention Score Altmetric é composto por mais de dez fontes distintas, como portais de notícias (imprensa), blogs, mídias sociais, gerenciadores de referência, dentre outros. A Tabela 1 apresenta a distribuição da média da atenção online da produção analisada pelas fontes mais expressivas (Mendeley, Twitter, Facebook, Imprensa e blogs). Fontes como Wikipédia, YouTube e Reddit foram desconsideradas, por conta dos baixos índices de incidência que apresentaram.

Tabela 3. Média de menções das áreas de conhecimento por tipo de fonte

\begin{tabular}{|l|l|l|l|l|l|}
\cline { 2 - 6 } \multicolumn{2}{c|}{} & Fontes \\
\hline Área do Estudo & Twitter & Facebook & Mendeley & Média & Blogs \\
\hline Ciências Agrárias & 3.85 & 1.55 & 10.45 & 3.50 & 1.37 \\
\hline Ciências Biológicas & 7.09 & 2.25 & 15.43 & 6.51 & 1.82 \\
\hline Ciências da Saúde & 12.61 & 2.80 & 14.38 & 11.37 & 2.02 \\
\hline Ciências Exatas e da Terra & 6.56 & 1.57 & 11.33 & 7.53 & 2.27 \\
\hline Ciências Humanas & 5.97 & 1.85 & 8.10 & 9.40 & 1.92 \\
\hline Ciências Sociais Aplicadas & 3.79 & 1.26 & 10.14 & 1.33 & 1.05 \\
\hline Engenharias & 3.51 & 1.22 & 11.80 & 3.57 & 2.05 \\
\hline Linguística, Letras e Artes & 3.29 & 1.29 & 3.70 & $\mathrm{NaN}$ & 1.00 \\
\hline Outras & 10.19 & 2.05 & 14.53 & 8.98 & 1.62 \\
\hline
\end{tabular}

Fonte: dados da pesquisa (2017)

O primeiro ponto a ser destacado é o bom desempenho do Mendeley, seguido do Twitter, e a baixa cobertura do Facebook, que muito embora seja a mídia social mais utilizada no Brasil, alcançou média de menções bem inferior às demais fontes, com exceção apenas dos blogs, cujo desempenho foi ainda menos significativo. Esse quadro parece repetir o resultado de pesquisas que obtêm dados da Altmetric.com, como a de Park e Park (2018), que analisou os indicadores altmétricos em países asiáticos, com frequência acentuada de artigos salvos no Mendeley, seguido de menções no Twitter e no Facebook. 
Esses resultados se assemelham também aos de outros estudos que analisaram a diferença de atenção online entre as fontes dos dados. Tanto na pesquisa de Priem, Piwowar e Hemminger (2012), que analisou dados altmétricos da coleção Public Library of Science (PLoS), quanto na de Alperin (2015), que verificou a atenção online da coleção Scientific Electronic Library Online (SciELO) de países da América Latina, e na de Travieso-Rodriguez e Araújo (2018) que investigou indicadores altmétricos de publicações de Brasil, Espanha e Portugal, os resultados identificaram maior cobertura de dados vindos do Mendeley, com média de leituras bem superior às menções no Twitter que, por sua vez, apresentou médias superiores às postagens no Facebook.

É interessante notar as diferenças de desempenho entre as áreas de conhecimento por tipo de fonte. O Mendeley, por exemplo, registra valores maiores nas Ciências Biológicas (15.43) - resultado similar ao estudo de Alperin (2015) - enquanto as Ciências da Saúde possuem maior cobertura no Twitter (12.61) e no Facebook (2.80). Outro destaque é o desempenho da imprensa, com médias que demonstram uma cobertura maior para as Ciências da Saúde (11.37), seguidas pelas Ciências Humanas (9.40), mas nenhuma cobertura para as áreas de Linguística, Letras e Artes. As áreas com melhor desempenho em blogs são as Ciências Exatas e da Terra (2.27) e as Engenharias (2.05).

Os resultados obtidos para as pesquisas brasileiras também diferem dos encontrados para os países asiáticos, analisados por Cho (2017), que observou uma presença mais significativa das ciências médicas no Twitter, embora os artigos das ciências sociais e das artes e humanidades sejam mais salvos no Mendeley.

Para Alperin (2015) as flutuações entre as áreas quanto aos níveis de cobertura são indicativas de diferentes usos de cada tipo de fonte, potencialmente representando práticas distintas em relação às mídias sociais e gestores de referências em cada área ou indicando que diferentes públicos estão sendo alcançados por cada disciplina.

\section{Considerações finais}

A produção brasileira dos bolsistas de produtividade do CNPq das várias áreas do conhecimento reflete as diferentes agendas de pesquisa e constitui corpo de conhecimento privilegiado sobre o pensamento dessas áreas no país, expressando compromissos com correntes teóricas, metodológicas e de pensamentos acerca de sua historicidade, atuação e tendências.

O estudo procurou avaliar essa produção sob a perspectiva de seu impacto social, aferido não pelas citações, mas pela atenção online que tais pesquisas despertam dentro e fora da comunidade científica ao serem mencionadas em mídias sociais. Estudos qualitativos são 
necessários para averiguar conteúdos e contextos dessas menções e aprimorar o quadro altmétrico da produção analisada.

Os resultados encontrados, seja de maneira geral, por idioma, área de conhecimento ou tipo de fonte, nos fazem questionar se os baixos índices de menções revelam uma cultura de não compartilhamento de informações científicas nas mídias sociais ou se indica, como apontado por Alperin (2015), uma questão de limitação e cobertura da ferramenta Altmetric, uma hipótese que precisa sempre ser considerada para análises do impacto altmétrico da produção científica nacional.

\section{Referências}

ALHOORI, H. et al. Altmetrics for Country-Level Research Assessment. In: TUAMSUK, K.; JATOWT, A.; RASMUSSEN, E. (ed.) The Emergence of Digital Libraries: research and practices. ICADL 2014. Lecture Notes in Computer Science, v. 8839, Springer, 2014.

ALPERIN, J. P.. Ask not what altmetrics can do for you, but what altmetrics can do for developing countries. Bulletin of the Association for Information Science and Technology, v. 39, n. 4, April/May 2013. Disponível em: $<$ https://onlinelibrary.wiley.com/doi/pdf/10.1002/bult.2013.1720390407 >. Acesso em: 07 ago. 2018.

ALPERIN, J. P.. Geographic variation in social media metrics: an analysis of Latin American journal articles. Aslib Journal of Information Management, v. 67, n. 3, p. 289-304, 2015. Disponível em: <https://doi.org/10.1108/AJIM-12-2014-0176 >. Acesso em: 30 set. 2018.

ALTMETRIC. How is the Altmetric score calculated? September, 2017. Disponível em: $<$ https://help.altmetric.com/support/solutions/articles/6000060969-how-is-the-altmetricattention-score-calculated >. Acesso em: 11 dez. 2017.

$\mathrm{CHO}$, J. A comparative study of the impact of Korean research articles in four academic fields using altmetrics. Performance Measurement and Metrics, v. 18, n. 1, p. 38-51, 2017. Disponível em: <https://doi.org/10.1108/pmm-02-2016-0005>. Acesso em: 30 set. 2018.

CONSELHO NACIONAL DE DESENVOLVIMENTO CIENTÍFICO E TECNOLÓGICO (CNPq). Bolsas no país. Produtividade em Pesquisa - PQ. 2017. Disponível em: <http://cnpq.br/apresentacao13/ >. Acesso em 11 dez., 2017.

COSTAS, R.; ZAHEDI, Z.; WOUTERS, P. Do "altmetrics" correlate with citations? Extensive comparison of altmetric indicators with citations from a multidisciplinary perspective. Journal of the Association of Information Science and Technology, v. 66, n. 10, July 2014. Disponível em: <http://dx.doi.org/10.1002/asi.23309 >. Acesso em 21 set., 2017.

DAVENPORT, H., BECK, J. The Attention Economy: Understanding the New Currency of Business. Boston, MA: Harvard Business School Press, 2001.

FONSECA, F. P. C.; DIGIAMPIETRI, L. A. Análise da Relação entre Obtenção de Bolsas de Produtividade do CNPq e Medidas Bibliométricas e de Análise de Redes Sociais. In: BRAZILIAN 
WORKSHOP ON SOCIAL NETWORK ANALYSIS AND MINING, n. 5, July 2016. Anais... Porto Alegre, RS: BraSNAM, 2016.

GOUVEIA, F. C. A altmetria e a interface entre a ciência e a sociedade. Trabalho Educação e Saúde, Rio de Janeiro, v. 14, n. 3, p. 643-645, dez. 2016. Disponível em: <http://dx.doi.org/10.1590/1981-7746-sip00126 >. Acesso em: 10 dez., 2017.

HUANG, W.; WANG, P.; WU, Q. A correlation comparison between Altmetric Attention Scores and citations for six PLOS journals. PLoS ONE, v. 14, n. 4, e0194962, 2018. Disponível em: <https://doi.org/10.1371/journal.pone.0194962>. Acesso em: 10 fev., 2019.

PARK, H.; PARK, H. W. Research evaluation of Asian countries using altmetrics: comparing South Korea, Japan, Taiwan, Singapore, and China. Scientometrics, v. 117, n. 2, p. 771-788, November 2018. Disponível em: <http://dx.doi.org/10.1007/s11192-018-2884-6 >. Acesso em: 21 set., 2018.

PERLIN, M. S.. Package GetLattesData: downloading and reading bibliometric data from Lattes. Set., 2017. Disponível em: <https://msperlin.github.io/2017-09-04-Package-GetLattesData/ >. Acesso em: 3 nov., 2017.

PRIEM, J.; PIWOWAR, H. A.; HEMMINGER, B. M. Altmetrics in the wild: Using social media to explore scholarly impact. ArXiv.org. 2012. Disponível em: <http://arxiv.org/abs/1203.4745 >. Acesso em 03 dez., 2017.

PRIEM, J. et al. Altmetrics: a manifesto. Out. 2010. Disponível em: $<$ http://altmetrics.org/manifesto >. Acesso em: 16 ago. 2016.

THELWALL, M.; MAFLAHI, N. Are scholarly articles disproportionately read in their own country? An analysis of Mendeley readers. Journal of the Association for Information Science and Technology, v. 66, n. 6, p. 1124-1135, June 2015. Disponível em: <https://onlinelibrary.wiley.com/doi/full/10.1002/asi.23252> Acesso em: 21 set. 2018.

TRAVIESO-RODRIGUEZ, C.; ARAÚJO, R. F. Indicadores altmétricos y de citación en la producción científica en ScienceOpen: estudio descriptivo para Brasil, España y Portugal. Bibliotecas Anales de investigación, v. 14, p. 124-137, 2018. Disponível em: <http://revistas.bnjm.cu/index.php/anales/article/view/4244/3897 > Acesso em: 21 set. 2018.

WAINER, J.; VIEIRA, P. Avaliação de bolsas de produtividade em pesquisa do CNPq e medidas bibliométricas: correlações para todas as grandes áreas. Perspectivas em Ciência da Informação, Belo Horizonte, v. 18, n. 2, p. 60-78, jun., 2013. Disponível em: <http://dx.doi.org/10.1590/S1413-99362013000200005 >. Acesso em 22 nov., 2017.

WANG, X. et al. The poor altmetric performance of publications authored by researchers in mainland China. Frontiers in Research Metrics and Analytics, v. 1, n. 8, October 2016. Disponível em: <http://dx.doi.org/10.3389/frma.2016.00008 >. Acesso em: 12 ago., 2018.

ZAHEDI, Z.; COSTAS, R.; WOUTERS, P. How well developed are altmetrics? A cross-disciplinary analysis of the presence of 'alternative metrics' in scientific publications. Scientometrics, v. 101, n. 2, p. 1491-1513, 2014. Disponível em: <https://doi.org/10.1007/s11192-014-1264-0 >. Acesso em: 12 ago., 2018. 